\title{
MacroH2A1 isoforms are associated with epigenetic markers for activation of lipogenic genes in fat-induced steatosis
}

\author{
Christine Podrini,* Apostolos Koffas,* Shilpa Chokshi,* Manlio Vinciguerra, ${ }^{\dagger}$ \\ Christopher J. Lelliott, ${ }^{\ddagger}$ Jacqueline K. White, ${ }^{\ddagger}$ Hibret A. Adissu, ${ }^{\S}$ Roger Williams, ${ }^{*}$ \\ and Azzura Greco*,1 \\ *Foundation for Liver Research, Institute of Hepatology, London, United Kingdom; ${ }^{\dagger}$ University College \\ London (UCL)-Institute for Liver \& Digestive Health, UCL Medical School, London, United Kingdom, \\ ${ }^{\ddagger}$ Mouse Genetics Project, Wellcome Trust Sanger Institute, Wellcome Trust Genome Campus, \\ Cambridge, United Kingdom; and ${ }^{\$}$ Physiology and Experimental Medicine Research Program, The \\ Hospital for Sick Children, University of Toronto, Toronto, Ontario, Canada
}

ABSTRACT The importance of epigenetic changes in the development of hepatic steatosis is largely unknown. The histone variant macroH2A1 under alternative splicing gives rise to macroH2A1.1 and macroH2A1.2. In this study, we show that the macroH2A1 isoforms play an important role in the regulation of lipid accumulation in hepatocytes. Hepatoma cell line and immortalized human hepatocytes transiently transfected or knocked down with macroH2A1 isoforms were used as in vitro model of fat-induced steatosis. Gene expressions were analyzed by quantitative PCR array and Western blot. Chromatin immunoprecipitation analysis was performed to check the association of histone H3 lysine 27 trimethylation (H3K27me3) and histone $\mathrm{H3}$ lysine 4 trimethylation (H3K4me3) with the promoter of lipogenic genes. Livers from knockout mice that are resistant to lipid deposition despite a high-fat diet were used for histopathology. We found that macroH2A1.2 is regulated by fat uptake and that its overexpression caused an increase in lipid uptake, triglycerides, and lipogenic genes compared with macroH2A1.1. This suggests that macroH2A1.2 is important for lipid uptake, whereas macroH2A1.1 was found to be protective. The result was supported by a high positivity for macroH2A1.1 in knockout mice for genes targeted by macroH2A1 (Atp5a1 and Fam $73 b$ ), that under a high-fat diet presented minimal lipidosis. Moreover, macroH2A1 isoforms differentially regulate the expression of lipogenic genes by modulating the association of the active (H3K4me3) and repressive (H3K27me3) histone marks on their promoters. This study underlines the importance of the replacement of noncanonical histones in the regulation of genes involved in lipid metabolism in the progression of steatosis.-Podrini, C., Koffas, A., Chokshi, S., Vinciguerra, M., Lelliott, C. J.,

Abbreviations: ChIP, chromatin immunoprecipitation; FFA, free fatty acid; GFP, green fluorescent protein; H3K4me3, histone H3 lysine 4 trimethylation; H3K27me3, histone H3 lysine 27 trimethylation; IHH, immortalized human hepatocytes; IP, immunoprecipitation; LPO, lipid peroxidation; ORO, Oil-Red Oil; siRNA, small interfering RNA; TBS-T, Trisbuffered saline with Tween; TSS, transcription start site
White, J. K., Adissu, H. A., Williams, R., Greco, A. MacroH2A1 isoforms are associated with epigenetic markers for activation of lipogenic genes in fat-induced steatosis. FASEBJ. 29, 1676-1687 (2015). www.fasebj.org

Key Words: histone methylation · bivalent promoter $\cdot$ histone variants $\cdot$ lipid uptake

THE RAPID INCREASE IN OBESTTY worldwide with unhealthy dietary patterns and sedentary lifestyles is associated with an increase in nonalcoholic fatty liver disease $(1,2)$. Nonalcoholic fatty liver disease is a pathologic condition that begins as simple steatosis but may further develop into nonalcoholic steatohepatitis and subsequently to advanced fibrosis, cirrhosis, and hepatocellular carcinoma (3-5). Hepatic steatosis is characterized by the accumulation of excess triglycerides resulting from an imbalance between synthesis, uptake, secretion, and oxidation of fatty acids (6). Although aberrant regulation of lipogenic genes is known to contribute to the development of hepatic steatosis, the molecular mechanisms involved in the regulation of lipogenic genes remain largely unclear (7). Recent studies support a role for epigenetic mechanisms including posttranslational modifications, changes in nucleosome positioning, and the replacement of canonical histones with their histone variants $(8,9)$. One such histone variant named macroH2A1 consists of a histone H2A-like domain fused to a large C-terminal nonhistone region called a macrodomain $(10,11)$. MacroH2A1 RNA can be alternatively spliced, giving rise to 2 different isoforms: macroH2A1.1 and macroH2A1.2, which differ in their macrodomain (12). Furthermore, macroH2A1 was reported to be enriched in the nucleosome of genes related to lipid metabolism, suggesting a role in the regulation of fatty acid uptake $(13,14)$. It has also been reported that mice fed

\footnotetext{
${ }^{1}$ Correspondence: Epigenetics of Fatty Liver Disease Research Group, Institute of Hepatology, Foundation for Liver Research, 69-75 Chenies Mews, London WC1E 6HX, United Kingdom. E-mail: a.greco@researchinliver.org.uk

doi: $10.1096 /$ fj.14-262717

This article includes supplemental data. Please visit http:// www. fasebj.org to obtain this information.
} 
a methyl-deficient diet showed considerable changes in the level of macroH2A1 (15). Moreover, we recently reported that the macroH2A1.2 isoform is up-regulated in a murine model of fat induced-hepatocellular carcinoma (16).

In the present study, we show that modulation of the expression of macroH2A1 isoforms induced by the accumulation of lipids is necessary to alter epigenetic modifications on the lipogenic gene promoters. We demonstrate that macroH2A1 isoforms act antagonistically to regulate lipid metabolism by affecting the state of active [histone $\mathrm{H} 3$ lysine 4 trimethylation (H3K4me3)] and inactive [histone H3 lysine 27 trimethylation (H3K27me3)] histone marks on the promoter of key lipogenic genes. Our findings provide new insight into histone modifications that, through the presence of macroH2A1, isoforms modulate the expression of genes involved in lipid metabolism and thereby have a direct influence on the development of hepatic steatosis.

\section{MATERIALS AND METHODS}

\section{Cell culture, transfection, and vectors}

Human hepatoma cell line HepG2 was obtained from American Type Culture Collection (ATCC, Manassas, VA, USA) and was cultured in DMEM supplemented with $10 \%$ fetal bovine serum and $100 \mathrm{U} / \mathrm{ml}$ penicillin/streptomycin solution (Invitrogen, Paisley, United Kingdom) at $37^{\circ} \mathrm{C}$ under $5 \% \mathrm{CO}_{2}$. Immortalized human hepatocytes (IHHs) isolated and immortalized by lentiviral transduction with the SV40T antigen and hTERT as described previously (17) were a kind gift from M.V. and cultured as previously described (18). Cells were grown to $70 \%$ confluence in 6/24-well plates and transiently transfected with pCQXIP-macroH2A1.1-Flag or pCQXIP-macroH2A1.2-Flag (kindly provided by Dr. Matthew Gamble, Albert Einstein College of Medicine, New York, NY, USA) (19) or empty green fluorescent protein (GFP)-pLNCX2 plasmids using Lipofectamine 2000 (Invitrogen) according to the manufacturer's instructions. Twenty-four hours after transfection, enhanced GFP was observed under a green fluorescent microscope, and the transfection efficiency was calculated based on the florescent intensity. On the same day ( $24 \mathrm{~h}$ after transfection), lipid accumulation was induced by incubating the cells with $0.2 \mathrm{mM}$ free fatty acids (FFAs) consisting of a 1:1 mix of oleic acid/linoleic acid (Sigma-Aldrich, Dorset, United Kingdom) for $24 \mathrm{~h}$ as described previously in the fat-induced steatosis model of Cui et al. (20). As a control, HepG2/IHH cells were incubated without addition of FFAs. After incubation, the extent of fat accumulation was determined by using $0.5 \%$ Oil Red-O solution (Sigma-Aldrich) in isopropanol for $10 \mathrm{~min}$. Subsequently, lipid droplets were examined with a fluorescent microscope (Leica Epi Fluorescence microscope; Leica Microsystems, Milton Keynes, United Kingdom) and quantified by NIH ImageJ software (National Institutes of Health, Bethesda, MD, USA).

\section{Quantitative RT-PCR}

Total RNA was extracted from HepG2/IHH cell lines by TRIzol isolation (Invitrogen) according to the manufacturer's instructions. cDNA was generated by using QuantiTect Reverse Transcription Kit (Qiagen, Manchester, United Kingdom). Quantitative PCR was performed using the SYBR Green (Applied Biosystems, Cheshire, United Kingdom) on an ABi7500 system. Gene expression data performed on key lipogenic genes [VLDLr, SCD,
FASN, FATP2, FATP4) were normalized to $\beta$-2-microglobulin and expressed as fold changes. Specific primers were designed: FATP2 forward 5'-TGGTGTCGCCAGAACTACAAG-3', reverse 5'-GAAAGAGTCAATCCCATCTGTGT-3'; FATP4 forward 5' CGGTTCTGGG-ACGATTGTAT-3', reverse 5'-AACCTGGTGCTGGTTTTCTG-3'; $\beta$-2-microglobulin forward $5^{\prime}$-TGAGTGCTGTCTCCATGTTTGA-3', reverse 5'-TCTGCTCCCCACCTCTAAGTTG-3'; macroH2A1.1 forward 5'-CCAGCCAAAAAGGCCAAG$3^{\prime}$ reverse 5'-CCCTGCTTCTTGGATTTCCG-3'; macroH2A1.2 forward 5'-CCAGCCAAAAAGGCCAAGTC-3' , reverse 5'-TCACCCTGCTTCTTCTTGGA-3' ${ }^{\prime}$ FASN forward $5^{\prime}$-AGGATGTCAACAAGCCCAAG-3', reverse 5'-ACAGAGGAGAAGGCCACAAA3'; Acyl-CoA synthetase long-chain family member 1 forward $5^{\prime}$ CTTCTGGTACGCCACGAGAC-3' , reverse 5'-GTCGCTGTCAAGTAGTGCG-3'; VLDLr forward 5' -TTCCAGTGCACAAATGGTCGCTGTA-3' ${ }^{\prime}$, reverse $5^{\prime}$-ATCACATCTCCAGGACACTGGGATA3'; SCD forward 5'-CGAGCCGGAGTTTACAGAAG-3', reverse 5'TATTTCCTCAGCCCCCTTTT-3'.

A quantitative PCR array of 86 fatty liver genes was designed and produced by SABiosciences (PAMM-157ZA) (SABiosciences, Manchester, United Kingdom). Samples were amplified by using SYBR green PCR 2X SABiosciences RT2 qPCR Master Mix (SABiosciences). Relative quantitation of gene expression was performed using the $2^{-\Delta \Delta \mathrm{Ct}}$ method and analyzed by using the manufacturer's software (http://pcrdataanalysis.sabiosciences.com/pcr/ arrayanalysis.php). To define differentially expressed genes, a filter consistent with statistical significance of $P<0.05$ and a fold expression change greater than 1.5 was applied.

\section{Western blot}

Protein extraction was performed by using RIPA buffer $(50 \mathrm{mM}$ Tris-HCl, pH 7.4, 1\% Nonidet P-40; 0.5\% sodium deoxycholate, $0.1 \% \mathrm{SDS}, 150 \mathrm{mM} \mathrm{NaCl}$ ). In brief, cells were suspended and homogenized in $200 \mu \mathrm{l}$ RIPA buffer and incubated on ice for $20 \mathrm{~min}$, followed by sonication. Samples were centrifuged at $13,000 \mathrm{~g}$ at $4^{\circ} \mathrm{C}$ for $30 \mathrm{~min}$. Twenty to $40 \mu \mathrm{g}$ of total protein from the cell lysate was subjected to electrophoresis in 4-12\% NuPAGE Bis-Tris Mini Gels (Invitrogen). Membranes were blocked for $30 \mathrm{~min}$ in $5 \%$ nonfat dry milk in Tris-buffered saline with Tween (TBS-T) buffer (1 M Tris-HCL, pH 8, $5 \mathrm{mM} \mathrm{NaCl}$, and $0.1 \%$ Tween-20) and subsequently blotted with the appropriate antibodies in TBS-T containing $5 \%$ nonfat dry milk. Antibodies were used at the following concentrations: anti-FLAG (1:1000) (f-7425) (SigmaAldrich), anti-macroH2A1.1 (1:100) (39871), anti-macroH2A1.2 (1:100) (61427), anti-H3K27me3 (1:2000) (39155), antiH3K4me3 (1:2500) (39159) (Active Motif, La Hulpe, Belgium); anti-FASN (1:500) (sc-48357) and anti-SCD (sc-30435) (1:100) (Santa Cruz Biotechnology, Heidelberg, Germany) were incubated overnight at $4^{\circ} \mathrm{C}$. Anti-actinin (1:5000) (sc-7454-R) (Santa Cruz Biotechnology) or anti-H3 (1:1000) (61475) (Active Motif) antibodies were used as loading controls. The membranes were incubated with the secondary antibodies at 1:3000 in TBS-T containing $5 \%$ nonfat dry milk for $1 \mathrm{~h}$. For immunoprecipitation (IP), the protein lysates were immunoprecipitated with anti-FLAG (3 $\mu \mathrm{g})$ (Sigma-Aldrich) overnight at $4^{\circ} \mathrm{C}$, and IgGs were used as negative controls. The FLAG-enriched lysates were subjected to electrophoresis in 4-12\% NuPAGE Bis-Tris Mini Gels (Invitrogen), and the binding was checked using antiH3K27me3, anti-H3K4me3 (Active Motif). Total lysates were used as input.

\section{FFA analysis}

FFA levels were measured by enzymatic assay (Sigma-Aldrich) according to the manufacturer's instructions. 


\section{Lipid peroxidation analysis}

Lipid peroxidation (LPO) levels were determined using a commercial lipid hydroperoxide assay kit from Calbiochem (Merck-Millipore, KGaA, Darmstadt, Germany) according to the manufacturer's instructions. Briefly, cellular homogenates $(500 \mu \mathrm{l})$ were deproteinated and extracted under acidic conditions with $1 \mathrm{ml}$ of ice-cold deoxygenated chloroform, and the extracts were removed following centrifugation $\left(1500 \mathrm{~g}\right.$ for $5 \mathrm{~min}$ at $0^{\circ} \mathrm{C}$ ) for LPO determination at the absorbance of $500 \mathrm{~nm}$.

\section{Immunofluorescence}

Immunofluorescence was performed as follows: cells were fixed in $4 \%$ paraformaldehyde for $5 \mathrm{~min}$ and permeabilized with $0.1 \%$ Triton X-100 (Sigma-Aldrich). Anti-FLAG (Sigma-Aldrich) (1:1000) primary antibody was incubated overnight at $4^{\circ} \mathrm{C}$. The staining was developed using Alexa fluorescent conjugated secondary antibodies, and images were acquired using a Leica Epi Fluorescence microscope.

\section{MacroH2A1 silencing}

Knockdown of macroH2A1 was performed by predesigned small interfering RNA (siRNA) directed against human H2AFY (SI04137749 and SI00433062) (Qiagen). HepG2 cells were transfected in 6-well plates with simacroH2A1 variants and nontarget siRNA as a negative control $(100 \mathrm{nM})$ for $48 \mathrm{~h}$ by using DharmaFect 1 (Invitrogen) at $60 \%$ confluence according to the manufacturer's instructions. This was followed by FFA treatment for $24 \mathrm{~h}$.

\section{Chromatin immunoprecipitation analysis}

Chromatin immunoprecipitation (ChIP) was performed using ChIP-IT or Re-ChIP Express Chromatin Immunoprecipitation Kit (Active Motif) according to the manufacturer's instructions. The chromatin was incubated with anti-H3K27me3, antiH3K4me3 (Active Motif) or rabbit normal IgG (sc-2027) (Santa Cruz). For the simultaneous presence of 2 proteins at the same genomic region, re-CHiP was performed. A sequential ChIP has been performed by using anti-Flag (Sigma-Aldrich) followed by anti-H3K27me3. The ChIP-enriched DNA samples were analyzed by quantitative PCR. PCR primers were designed to locate the transcription start site (TSS) of the FASN or SCD promoters. A set of primers (8 primers for $\mathrm{SCD}$ and 5 for FASN) were designed to span the promoter region of FASN or SCD $(\sim 1 \mathrm{~kb})$. Primers are available on request. The most significant enrichment with H3K27me3/H3K4me3 on the FASN and SCD promoters was found on the following regions: -967 to -785 from the TSS of the FASN promoter forward 5'-TGTGGTGTGTGGGTTGGTAT-3', reverse 5'-AGGCTATCCCCATTTCGTCC-3'; and +42 to +179 from the TSS of the SCD promoter forward $5^{\prime}$-AGCTCTCATGGTAAGGCTCC-3', reverse 5'-CAGTTGAGCCCAGAAACACC-3'. Results are represented as fold enrichment after normalization with the DNA input.

\section{Animals and histopathology}

Mice carrying knockout first conditional-ready alleles (21) for Atp5a1 (Atp5a1 ${ }^{\text {tm1a(EUCOMM)Wtsi } / A t p 5 a 1^{+} \text {heterozygous animals) }}$ or Fam73b (Fam73b $b^{\text {tm1a(KOMP)Wtsi }} /$ Fam $73 b^{\text {tm1a(KOMP)Wtsi }}$ homozygous animals) were generated on a C57BL/ 6 genetic background by the Mouse Genetics Project (22). Genotyping, allele quality control, and phenotyping were performed as described previously (23). In brief, at $4 \mathrm{wk}$ of age, mice were transferred from Mouse Breeders Diet (LabDiet 5021-3; IPS, Richmond, VA, USA) to a high-fat ( $21.4 \%$ fat by crude content; $42 \%$ calories provided by fat) dietary challenge (Special Diet Services Western RD 829100; SDS, Witham, United Kingdom). At 16 wk of age, mice ( $n=2$ male and 2 female mice per mutant strain, plus matched controls) were humanely killed, and a full necropsy was performed, including collection of the liver into $10 \%$ neutral buffered formalin. Liver samples was fixed between 15 and $20 \mathrm{~h}$, processed overnight (Tek VIP 5; Sakura, Thatcham, United Kingdom), embedded in paraffin, and sectioned at $4 \mu \mathrm{m}$ for routine hematoxylin and eosin staining (24). All experiments were performed according to protocols approved by the UK Home Office regulations, UK Animals (Scientific Procedures) Act of 1986.

\section{Immunohistochemistry}

Liver sections were deparaffinized in xylene and rehydrated, and antigen retrieval treatment was carried out in $2 \mathrm{~N} \mathrm{HCl}$ buffer, $\mathrm{pH}$ 2 (preheated to $98^{\circ} \mathrm{C}$ ). Sections were then cooled, rinsed, and endogenous peroxidase blocked for 20 min with $0.5 \% \quad \mathrm{H}_{2} \mathrm{O}_{2}$. Finally, the sections were washed in double-distillated water followed by Tris-buffered saline with $0.01 \%$ Triton X-100 and then rinsed. Mouse liver sections were incubated with antimacroH2A1.2 and anti-macroH2A1.1 (Active Motif) 1:100 overnight at $4^{\circ} \mathrm{C}$. Sections were rinsed and incubated with the biotinylated antibody (Vector Laboratories, Peterborough, United Kingdom) and revealed with the 3,3'-diaminobenzidine horseradish peroxidase substrate kit (Vector Laboratories). The sections were finally dehydrated and coverslipped. Samples processed without primary antibodies served as negative controls. Five digital images from nonoverlapping fields were taken, and the number of positive nuclei was calculated.

\section{Statistical analysis}

All results are expressed as means \pm SEM. The statistical significance of differences in mean values was assessed by Student $t$ test for the 2 groups. All experiments were performed in triplicate and repeated 3 times independently unless otherwise stated.

\section{RESULTS}

\section{Role of macroH2A1 isoforms in an in vitro model of steatosis}

To understand whether FFA differentially influences the expression of the 2 isoforms, HepG2 and IHH cells were used as an in vitro model of steatosis (20). In this study, the mRNA and protein expression levels of endogenous macroH2A1.1 were unchanged, whereas that for macroH2A1.2 were significantly induced by FFA treatment (Fig. 1A), suggesting that the macroH2A1.2 is the isoform regulated by fat-induced steatosis.

The transient overexpression of macroH2A1.1 in the presence of FFA resulted in reduced level of lipids compared with the cells transfected with macroH2A1.2 and the empty GFP control vector (Fig. $1 B$ ). Quantitative analysis showed a significant decrease $(P<0.01)$ of the average droplets size and average total droplet surface area per cell for macroH2A1.1 compared with the macroH2A1.2-overexpressing cells and the control in HepG2 (Fig. 1C) and IHH cells in the presence of FFAs (Supplemental Fig. S1A).

To distinguish between intracellular redistribution of fat from true lipid accumulation, we measured the total amount of triglycerides in the cells transfected with macroH2A1.1 or macroH2A1.2 vs. cells transfected with the 


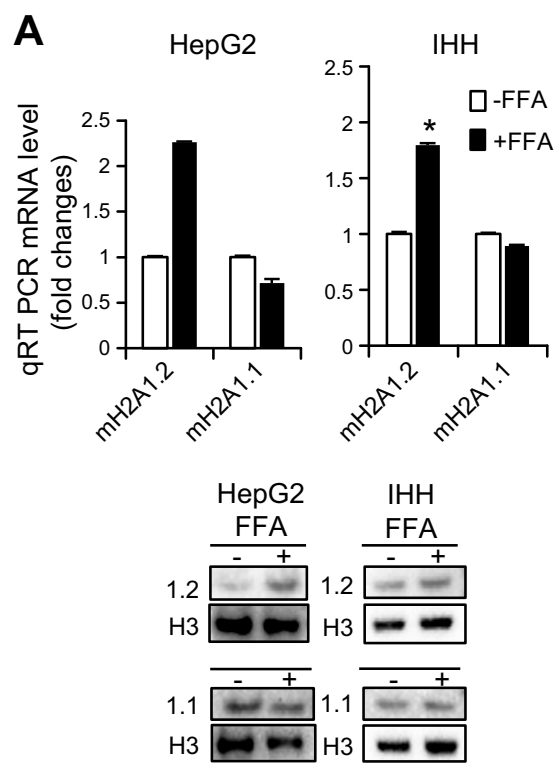

B

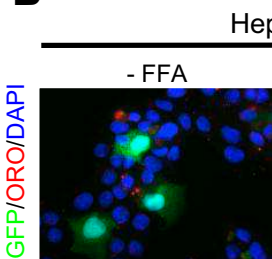

HepG2
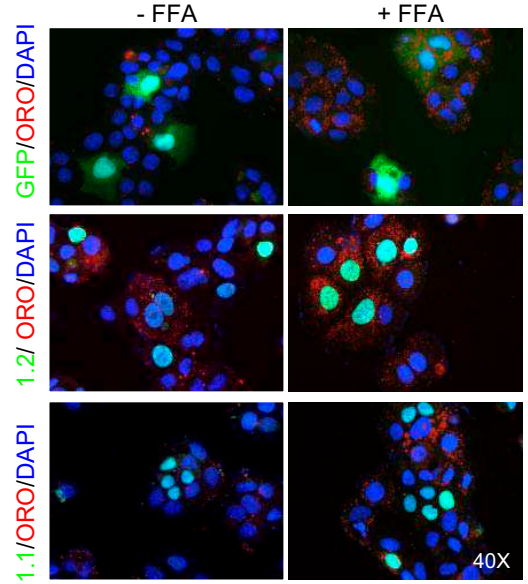

$\mathrm{IHH}$

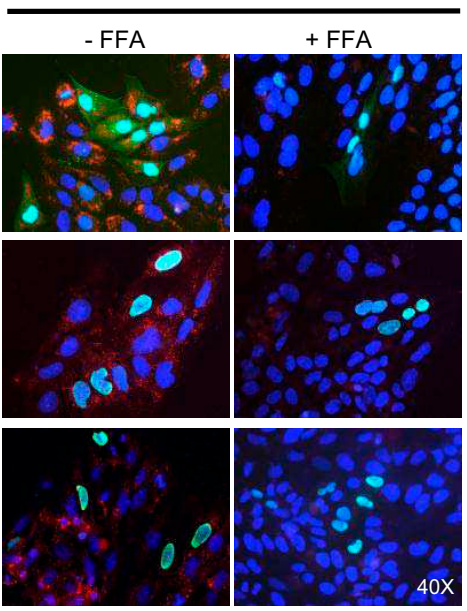

C

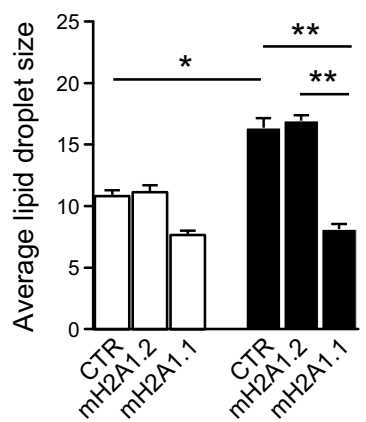

$\mathbf{E}$

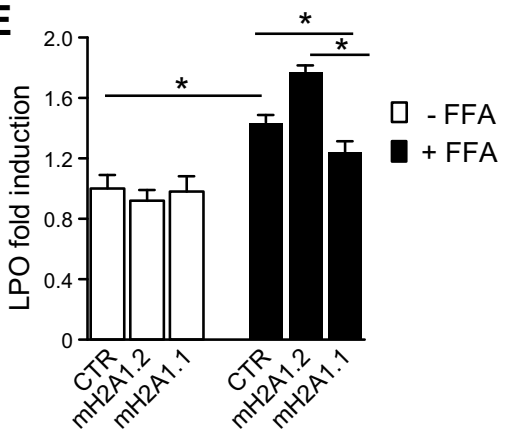

D

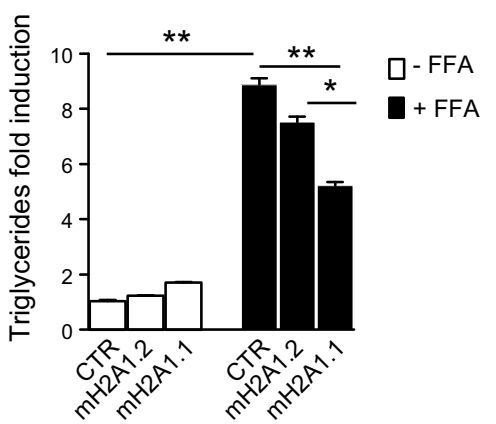

$\mathbf{F}$

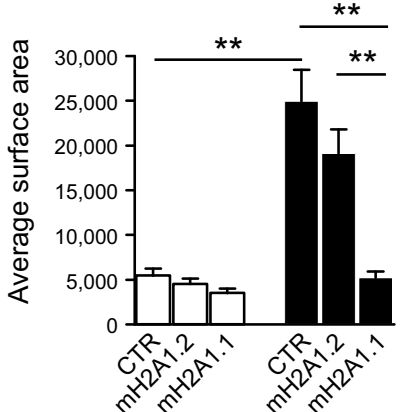

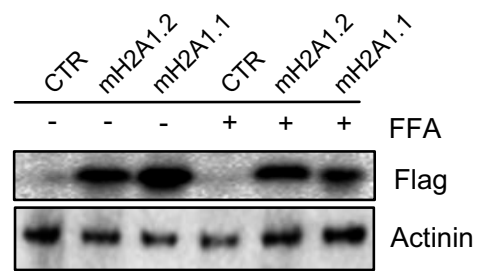

Figure 1. macroH2A1 isoforms regulate lipid uptake. A) (Left) Relative mRNA levels of macroH2A1 isoforms in HepG2 and IHH cells \pm FFA, examined by real-time PCR. Values for control were set at 1 . Right) Western blot analysis of macroH2A1 isoforms in HepG2 and IHH cells. H3 was used as a loading control. B) Immunofluorescence of HepG2 and IHH overexpressing macroH2A1 isoforms and empty vector GFP as a control. Showing macroH2A1-Flag-tagged in green, lipid droplets stained with ORO (red) and DAPI (blue) for nuclear stain with original magnification, $\times 40$. Representative pictures of 3 independent experiments. C) Quantification of individual lipid droplet size per surface area in $>100$ cells using ImageJ software. $* P<0.05$, $* * P<0.01 ; n=3 . D)$ Triglyceride measurement in HepG2-overexpressing cells. Values are normalized with the amount of protein. $* P<0.05, * * P<0.01 ; n=3$. E) Lipid peroxidation measurement in HepG2-overexpressing cells. Values are normalized with the amount of protein. Error bars represent SEM. $* P<0.05$, $* * P<0.01 ; n=3$. Results are represented as means \pm SEM of $\geq 3$ independent experiments. $F$ ) Western blot analysis of macroH2A1 overexpressing in HepG2 cells \pm FFA for anti-Flag and actinin as loading control.

control vector. The level of triglycerides was significantly reduced $(P<0.01)$ in cells overexpressing macroH2A1.1 compared with the control and macroH2A1.2 in the presence of FFA in HepG2 (Fig.1D) and IHH cells (Supplemental Fig. $\mathrm{S} 1 B)$.
Because excess FFAs were previously suggested to trigger lipid peroxidation, a signature of cellular damage, in cultured hepatocytes (25), we measured the formation of lipid peroxides in macroH2A1.1 or 1.2 overexpressing cells in the presence of FFAs. It was found 
that macroH2A1.1-overexpressed cells decreased lipid peroxidation significantly $(P<0.05)$ in the presence of FFA compared with the control and macroH2A1.2 in the presence of FFAs (Fig. 1E). These observations suggest that macroH2A1 isoforms have a distinct and opposite role in lipid uptake, with macroH2A1.1 playing a protective role in lipid uptake compared with macroH2A1.2.

\section{Lipid metabolic genes regulated by macroH2A1 isoforms}

A quantitative PCR array profile of the genes involved in lipid accumulation was performed to gain insight into genes and pathways modulated by the macroH2A1 isoforms in HepG2 cells.

The expression of key genes, involved in lipid accumulation and hepatic insulin resistance, was measured by comparing the control cells without FFAs vs. controls cells and macroH2A1-overexpressed isoforms with FFAs. The presence of FFAs caused an up-regulation of genes involved in lipid metabolism (Table 1). This was also confirmed in IHH cells for key lipogenic genes (Supplemental Fig. S2A).

Hierarchical clustering of the expression data revealed similar profiles in the control and macroH2A1.2overexpressing cells with addition of exogenous FFAs. This similar response might be caused by the endogenous upregulation of macroH2A1.2 in the presence of FFAs. Overexpression of macroH2A1.1 resulted in a minimal gene expression changes (Fig. 2A). Overall, a set of 36 genes, involved in lipid synthesis, glucose uptake, and $\beta$-oxidation, was found to be up-regulated in macroH2A1.2 and control cells after FFA administration compared with macroH2A1.1-overexpressing cells (Table 1).

TABLE 1. Gene expression analysis in macroH2A1-overexpressing cells

\begin{tabular}{|c|c|c|c|c|c|c|}
\hline \multirow[b]{2}{*}{ Gene name } & \multicolumn{2}{|c|}{ CTR FFA } & \multicolumn{2}{|c|}{ macroH2A1.2 FFA } & \multicolumn{2}{|c|}{ macroH2A1.1 FFA } \\
\hline & $\begin{array}{c}\text { Fold } \\
\text { change }\end{array}$ & $P$ value & $\begin{array}{c}\text { Fold } \\
\text { change }\end{array}$ & $P$ value & $\begin{array}{c}\text { Fold } \\
\text { change }\end{array}$ & $P$ value \\
\hline \multicolumn{7}{|c|}{ Lipid metabolism/transport } \\
\hline APOA1 & 3.42 & 0.0002 & 2.18 & 0.00065 & 1.33 & 0.0020 \\
\hline ACADL & 1.81 & 0.0110 & 2.27 & 0.0151 & 0.98 & 0.1680 \\
\hline SREBF1 & 1.62 & 0.0110 & 1.53 & 0.0294 & 0.74 & 0.0340 \\
\hline SREBF2 & 1.76 & 0.0050 & 2.26 & 0.0072 & 0.21 & 0.0413 \\
\hline HNF4A & 1.69 & 0.0220 & 1.50 & 0.0350 & 0.36 & 0.0055 \\
\hline APOC3 & 3.07 & 0.0010 & 2.60 & 0.0063 & 1.21 & 0.0496 \\
\hline PPARGC1A & 1.54 & 0.0003 & 1.69 & 0.0020 & 1.2 & 0.0002 \\
\hline SCD & 1.73 & 0.0129 & 2.37 & 0.0051 & 1.31 & 0.0438 \\
\hline CNBP & 1.79 & 0.0526 & 1.21 & 0.1310 & 1.04 & 0.3420 \\
\hline CYP7A1 & 2.08 & 0.0446 & 1.80 & 0.0178 & 1.44 & 0.0451 \\
\hline APOE & 1.09 & 0.2140 & 1.58 & 0.0226 & 1.21 & 0.0040 \\
\hline LDLR & 1.45 & 0.0460 & 1.63 & 0.0220 & 0.97 & 0.4979 \\
\hline FABP1 & 1.73 & 0.0038 & 3.63 & 0.0023 & 1.42 & 0.0009 \\
\hline FABP2 & 1.13 & 0.0001 & 1.73 & 0.0186 & 1.01 & 0.3972 \\
\hline CD36 & 1.66 & 0.0357 & 1.68 & 0.0186 & 1.05 & 0.3972 \\
\hline PNPLA3 & 0.44 & 0.0177 & 1.53 & 0.0319 & 1.45 & 0.0345 \\
\hline XBP1 & 0.89 & 0.3180 & 0.79 & 0.0700 & 0.05 & 0.1800 \\
\hline \multicolumn{7}{|c|}{$\begin{array}{l}\text { Glucose transporters, } \\
\text { gluconeogenesis, insulin } \\
\text { resistance, and inflammation }\end{array}$} \\
\hline SLC2A1 & 1.97 & 0.0477 & 2.24 & 0.0121 & 1.39 & 0.0084 \\
\hline SLC2A2 & 1.86 & 0.0312 & 2.03 & 0.0082 & 1.15 & 0.0009 \\
\hline SLC2A4 & 1.61 & 0.0040 & 1.54 & 0.0280 & 1.12 & 0.0005 \\
\hline G6PC & 2.16 & 0.0090 & 3.24 & 0.0050 & 1.56 & 0.0480 \\
\hline GCK & 1.57 & 0.1100 & 1.78 & 0.0230 & 1.40 & 0.0003 \\
\hline AKT1 & 2.06 & 0.0115 & 2.04 & 0.0006 & 0.99 & 0.4420 \\
\hline IRS1 & 1.80 & 0.0013 & 1.75 & 0.0001 & 0.62 & 0.0450 \\
\hline IL6 & 1.45 & 0.0130 & 2.67 & 0.0071 & 2.50 & 0.0146 \\
\hline MAPK1 & 0.98 & 0.1030 & 1.50 & 0.0358 & 1.03 & 0.2738 \\
\hline FOXA2 & 0.70 & 0.0015 & 0.93 & 0.3820 & 0.46 & 0.0567 \\
\hline IFNG & 3.18 & 0.0003 & 1.16 & 0.0239 & 2.14 & 0.2002 \\
\hline IGFBP1 & 0.34 & 0.0079 & 2.53 & 0.0040 & 1.19 & 0.0001 \\
\hline TNFRSF6 & 2.52 & 0.0950 & 1.53 & 0.0365 & 1.94 & 0.1345 \\
\hline \multicolumn{7}{|c|}{$\beta$-Oxidation and lipogenesis } \\
\hline ABCA1 & 1.54 & 0.0161 & 1.97 & 0.01740 & 1.20 & 0.127 \\
\hline ATP5C1 & 1.94 & 0.0132 & 1.99 & 0.01130 & 0.92 & 0.405 \\
\hline PPA1 & 1.75 & 0.0139 & 1.80 & 0.02660 & 0.97 & 0.443 \\
\hline NDUFB6 & 1.70 & 0.0482 & 1.87 & 0.01530 & 0.92 & 0.462 \\
\hline PPARG & 1.32 & 0.0261 & 1.58 & 0.03040 & 0.84 & 0.210 \\
\hline FASN & 2.36 & 0.0002 & 2.90 & 0.00450 & 1.11 & 0.270 \\
\hline
\end{tabular}




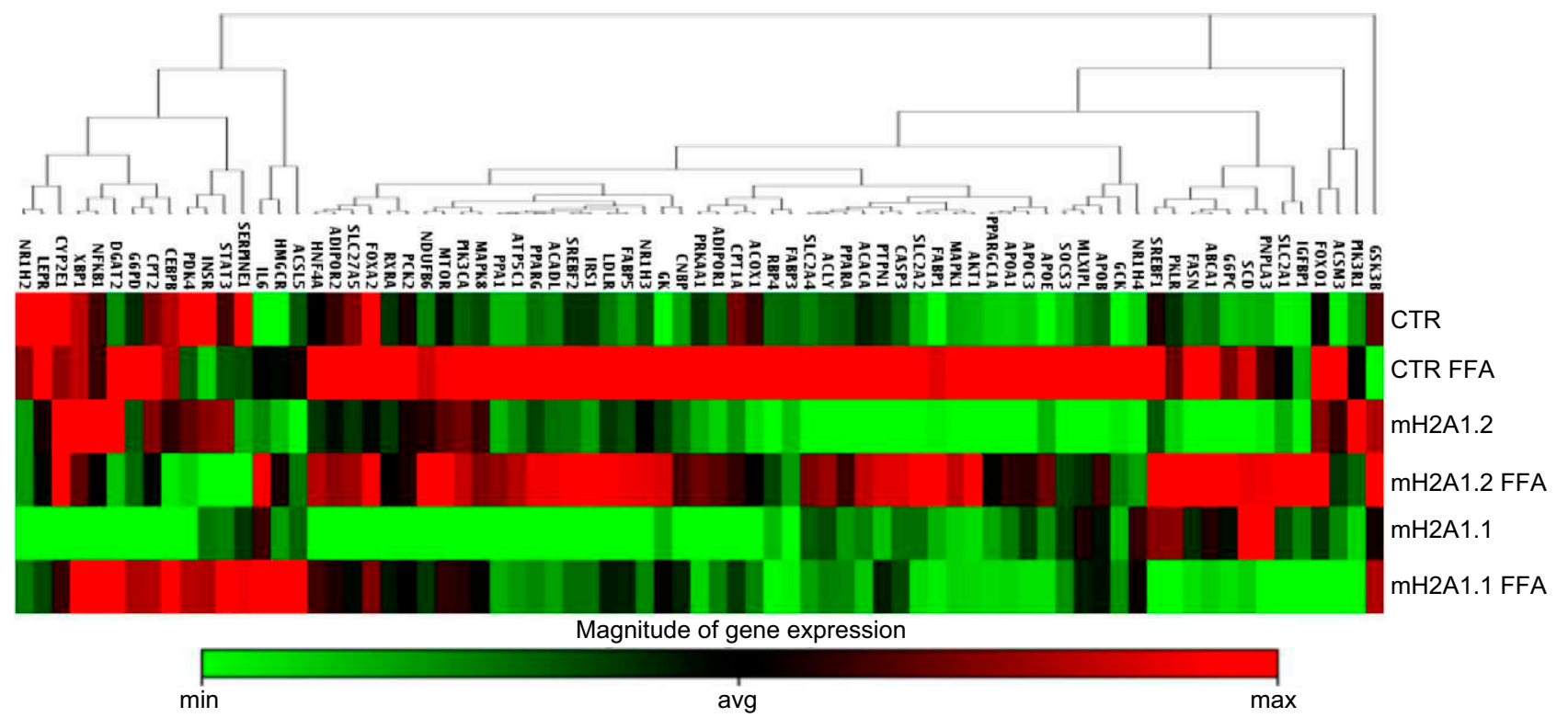

Figure 2. Heat map profile of fatty liver genes regulated by macroH2A1 isoforms in HepG2-overexpressing cells in control, macroH2A1.2, and macroH2A1.1 \pm FFA treatment. The graphic color of each gene is assigned on the basis of the measured signal ratio to the housekeeping genes GADPH, ACTB, and $\beta$-2-microglobulin. Expression levels are represented in a color scale from green (low expression) to red (high expression). Results are represented as means of $\geq 3$ independent experiments.

MacroH2A1.1-overexpressing cells in the presence of FFAs did not significantly induce the expression of genes involved in lipogenesis (FASN; $P>0.05$ ) and genes involved in gluconeogenesis (G6PC and GCK; $P<0.05$ ) compared with macroH2A1.2 cells. This shift from fatty acid oxidation to de novo lipid synthesis was mediated by a decreased activity of sterol regulatory element-binding transcription factor1/2 (SREBF1, SREBF2; $P<0.05$ ). SREBF1/2, master regulators of lipogenic genes such as SCD and FASN (26), were found to be down-regulated $(P<0.05)$ in FFA treatment. Further, the comparison between control with FFAs vs. macroH2A1.1-overexpressing cells in the presence of FFAs showed an overall downregulation of the lipogenic genes (Supplemental Table S1).

On the contrary, overexpression of macroH2A1.2 resulted in the up-regulation of genes involved in gluconeogenesis (G6PC and GCK; $P<0.05)$ and glucose transporters (SLC2A1, SLC2A2, and SLC2A4; $P<0.05$ ). Moreover, the expression of genes related to de novo lipogenesis (PPAR $\gamma ; P<0.05$ ) and $\beta$-oxidation (ABCA1, ACADL, ATP5C1, PPA1, and NDUFB6; $P<0.05$ ) were also increased, as well as genes involved in hepatic insulin resistance (AKT1 and IRS1; $P<0.01$ ).

To further explore the direct role played by macroH2A1 isoforms in lipid metabolism, studies were carried out using siRNA-mediated gene silencing as an experimental approach to generate a cellular model deficient in total macroH2A1 (Fig. 3A). The knockdown of macroH2A1 showed a reduction of lipid accumulation by Oil-Red Oil (ORO) staining in HepG2 (Fig. 3B, C) and IHH (data not shown) cells, concomitant with a down-regulation of mRNA expression for the fatty acid transporters (FATP2 and FATP4) and key lipogenic genes (SCD, FASN, and VLDLr) (Fig. 3D). On macroH2A1 knockdown, the expression of these genes remained down-regulated, even after FFA treatment, at the mRNA (Fig. 3D) and protein levels
(Fig. 3E). These observations raised the possibility that macroH2A1 is fundamental in the regulation of key lipogenic genes and that the isoforms play an antagonistic role in the activation and/or repression of lipogenic genes.

\section{Genetically induced mouse models fed a high-fat diet present high positivity of macroH2A1.1 isoform}

Previous studies have shown that several genes, belonging to ATP-dependent membrane transporters $(A t p)$ and family with sequence similarity members $(\mathrm{Fam})$, are targeted by macroH2A1 (27). Atp family members encode proteins that have direct or inferred connections with the transport of lipids (28-30). Atp5a1 is a component of ATP synthase complex (31), and Fam 73 b encodes a conserved membrane protein of unknown function, but recent evidence suggests that Fam73b has been associated to defects of bone strength and mineralization (32). Recent evidence further suggests that disruption of the genes Atp5a1 and Fam $73 b$ cause metabolic defects (22). Specifically, the absence of Atp5a1 decreases the efficiency of ATP generation via the oxidative phosphorylation route, suggesting that alternative mechanisms such as glycolysis will be upregulated and may cause a lean phenotype (33).

To better characterize the role of macroH2A1 in the development of steatosis, we checked the expression of the 2 isoforms in genetically modified mice null for Atp5a1 and Fam73b. Interestingly, both lines presented with a lean phenotype, decreased body weight, and decreased body fat (22). Despite high-fat diet consumption, and consistent with the observed clinical phenotypes, both lines presented minimal or absent lipidosis (Fig. $4 \boldsymbol{A}$ ). In this context, we checked the expression of macroH2A1 isoforms. The immunohistochemistry analysis showed high positivity for the macroH2A1.1 isoform $(P<0.01)$ compared with 
B

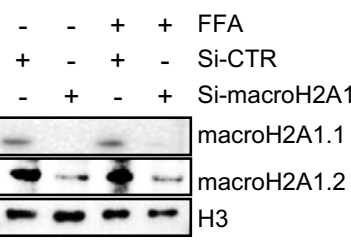

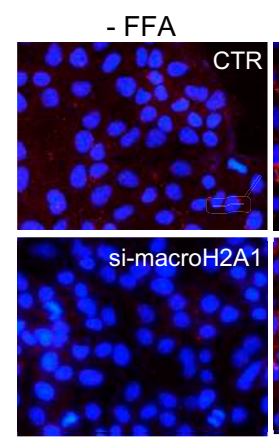
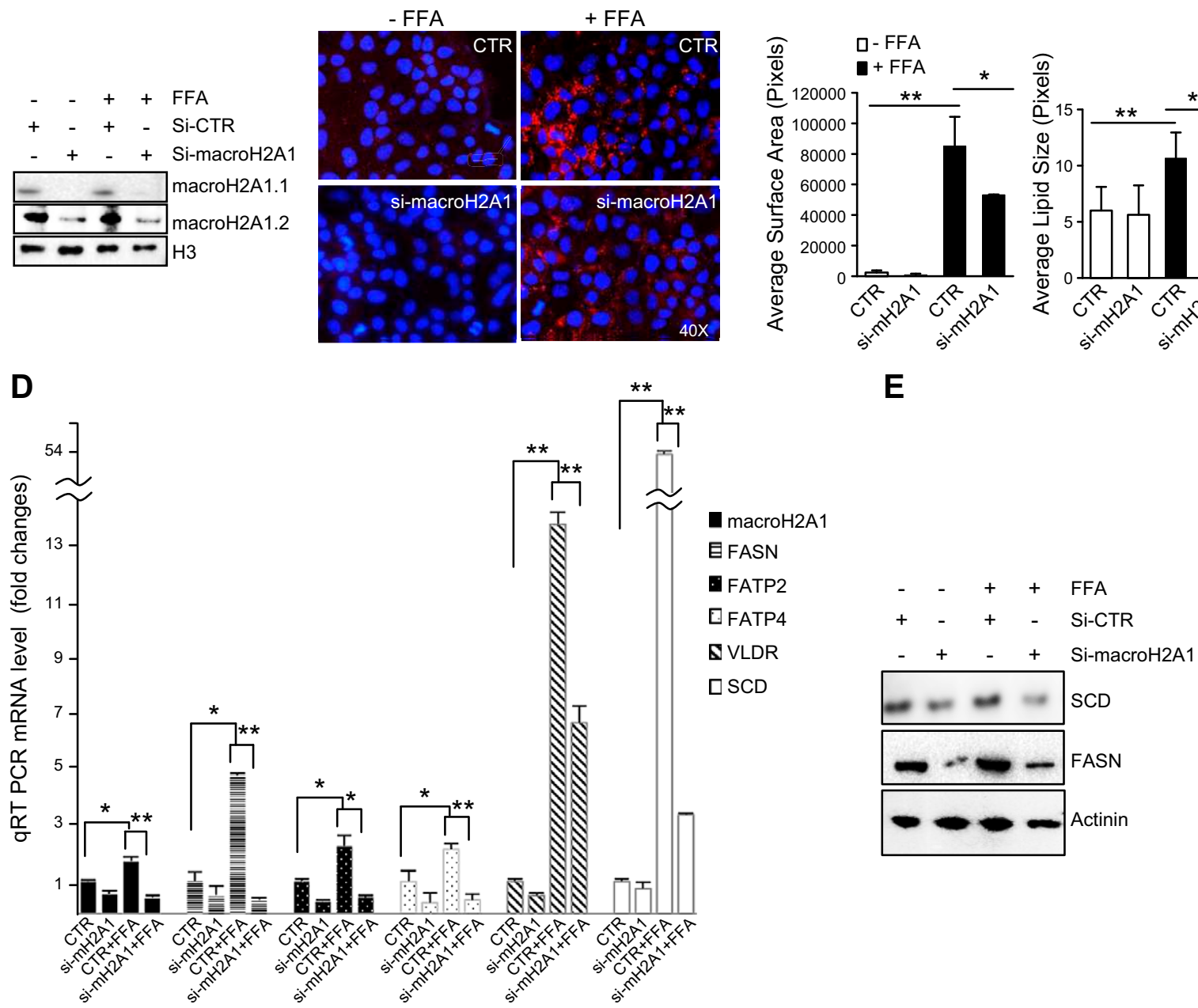

Figure 3. Lipid accumulation and gene expression in macroH2A1 knockdown in HepG2 cells. A) Protein expression level in simacroH2A1 isoforms \pm FFA. B) Immunofluorescence showing ORO (lipid stain) in simacroH2A1 \pm FFA. C) Quantification of individual lipid droplet size per surface area in $>100$ cells using ImageJ software. $* P<0.05, * * P<0.01 ; n=3$. D) Relative lipogenic gene mRNA levels in simacroH2A1 HepG2 \pm FFA. Values for control were set at 1 . Error bars represent SEM. $* P<0.05$, $* * P<0.01 ; n=3$. E) Western blot analysis of FASN and SCD in simacroH2A1 HepG2 \pm FFA, and actinin was used a loading control.

macroH2A1.2 (Fig. 4B). This suggests that the high positivity for the macroH2A1.1 isoform is related in vivo to the protection of the hepatocytes from a fat-induced model of steatosis.

\section{macroH2A1 isoforms affects the expression of epigenetic marks}

MacroH2A1 is a repressive epigenetic mark and is highly associated with trimethylation of H3K27me3. However, macroH2A1 may play a positive role in gene expression in a subset of genes when it is bound in transcribed regions (34). In the in vitro model of steatosis, we found that FFAs induced H3K27me3, a transcriptional repressive histone code, and increased level of trimethylation of H3K4me3, a transcriptional active histone code. The silencing of macroH2A resulted in a reduced expression of both repressive and active histone marks that were maintained even in the presence of exogenous FFA, suggesting that both histone marks are strongly associated with the presence of macroH2A1. Moreover, the overexpression of the
macroH2A1.2 isoform not only restored the expression of the histone marks, but also of the lipogenic genes (FASN and SCD) (Fig. 5A). This was also confirmed by quantitative PCR (Supplemental Fig. 3).

Next, IP showed that H3K27me3 and H3K4me3 were strongly associated with both macroH2A1 isoforms, suggesting that macroH2A1 can affect chromatin compaction (Fig. $5 B$ ) by the modulation of the histone marks.

\section{macroH2A1 isoforms affect epigenetic histone marks of lipogenic promoters}

Because SCD and FASN expression was differentially regulated by the macroH2A1 isoforms in the presence of FFAs, we further investigated whether macroH2A1 can affect the expression of SCD and FASN by altering the level of the 2 transcriptionally active (H3K4me3) and inactive (H3K27me3) histone marks.

ChIP analysis showed that in the control HepG2 cells, the level of H3K4me3 was increased, whereas the 


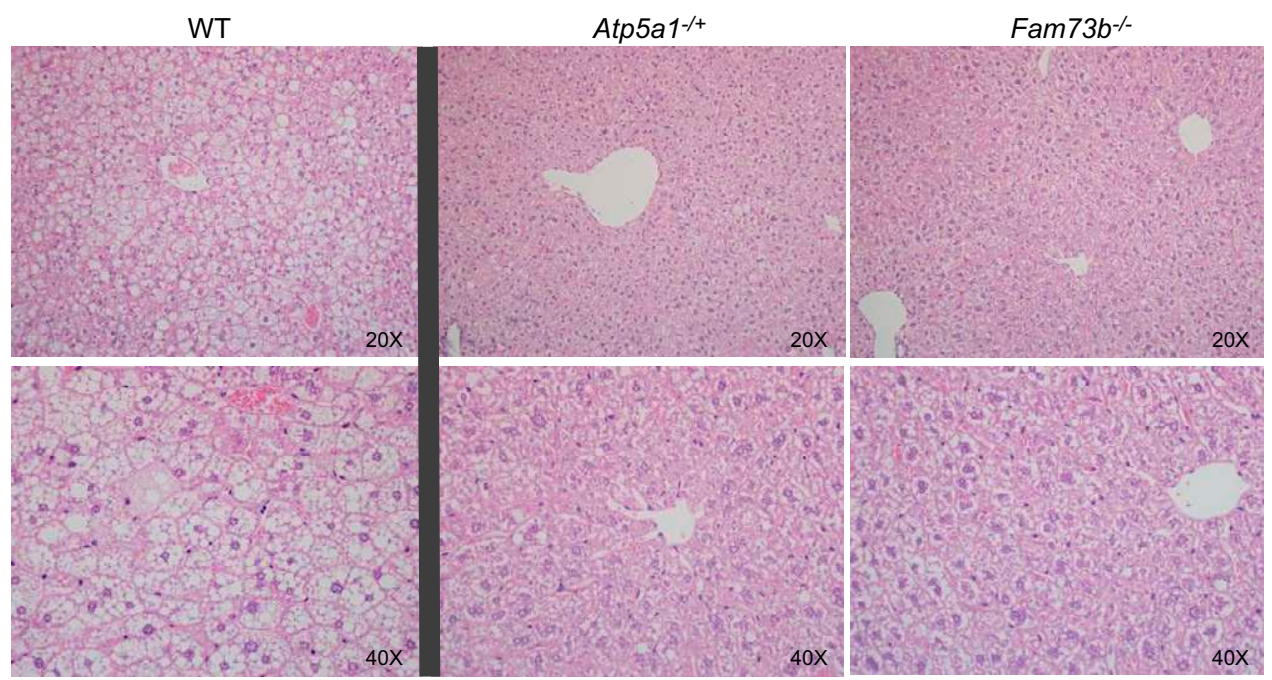

B
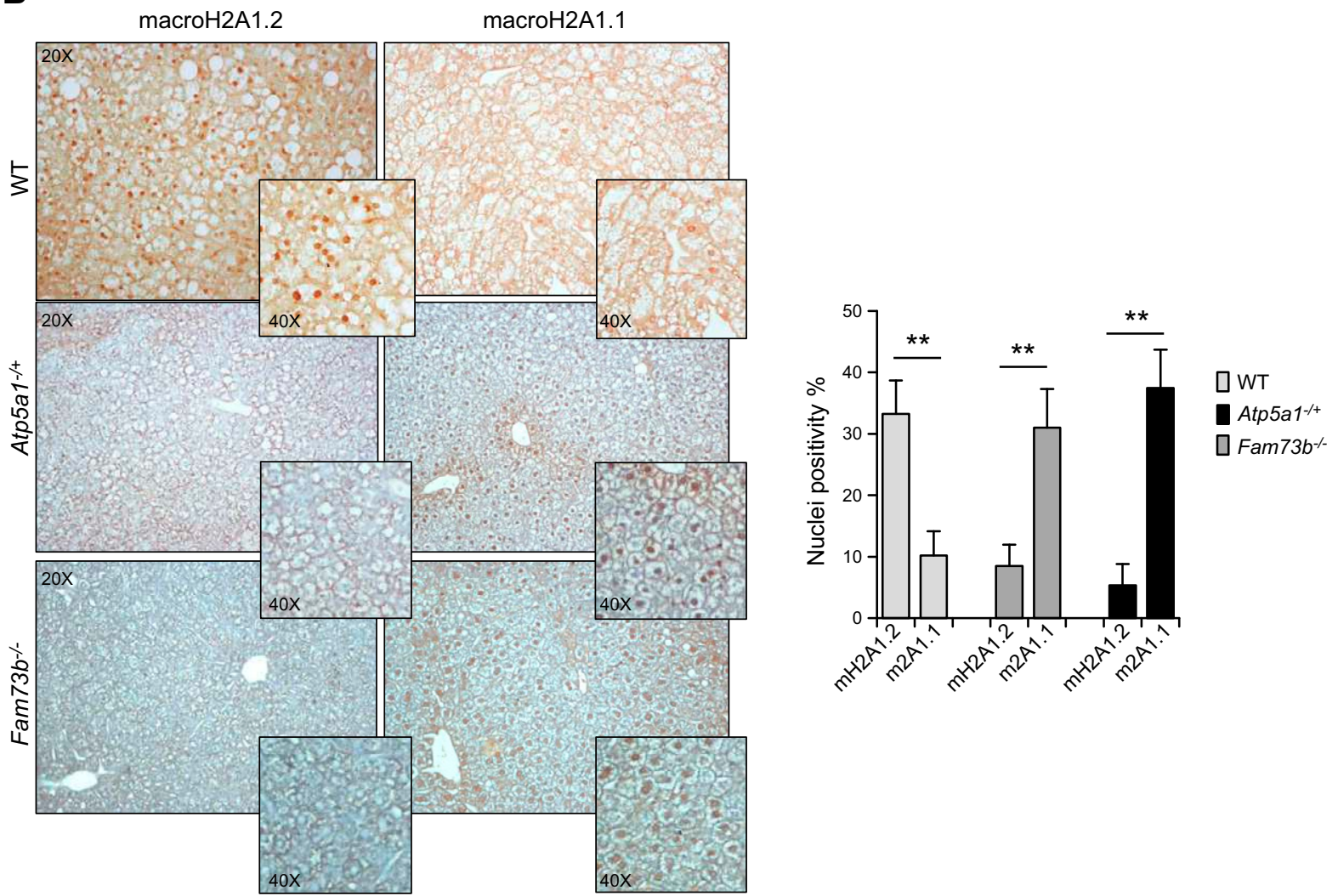

Figure 4. Absent or minimal hepatic lipidosis in $A t p 5 a 1^{-/+}$and $F a m 73 b^{-/-}$mice is associated with differential expression of macroH2A1.1 and macroH2A1.2. A) Histopathology revealed absent or minimal hepatic lipidosis in Atp5a1 $1^{-/+}$and Fam $73 b^{-/-}$ mice on a high-fat diet in contrast to the severe hepatic lipidosis in wild-type mice on the same diet. $B$ ) Minimal or absent hepatic lipidosis in Atp5a1 $1^{-/+}$and $\mathrm{Fam}_{73 b^{-/-}}$mice was accompanied by increased macroH2A1.1 and decreased macroH2A1.2 expression in hepatocyte nuclei compared with wild-type mice on a high-fat diet. Bar graph shows the percentage of hepatocyte nuclei that were positive for macroH2A1.1 and macroH2A1.2 in Atp5a1 $1^{-1+}$ and Fam $73 b^{-/-}$mice. $* * P<0.01 ; n=2$ male and 2 female mice per mutant strain, plus matched controls.

repressive histone mark H3K27me3 was decreased on the SCD and FASN promoter in the presence of FFA. Interestingly, SCD and FASN promoters showed high levels of both transcriptional marks after the knockdown of macroH2A1, suggesting that SCD and FASN promoters are retained in a bivalent state that can change in either direction to an open or closed state (lack of expression) under a specific stimuli. However, the reoverexpression of macroH2A1 isoforms resolved the FASN and SCD bivalent promoters toward an active histone code H3K4me3 when macroH2A1.2 is reoverexpressed and toward a repressed histone code H3K27me3 when macroH2A1.1 is reoverexpressed (Fig. 6A). Further, macroH2A1 isoforms and the epigenetic mark (H3K27me3) were sequentially 


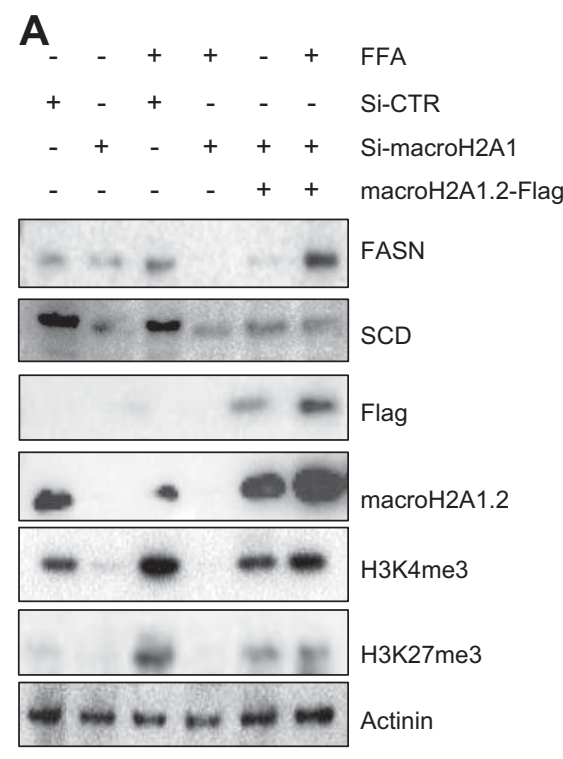

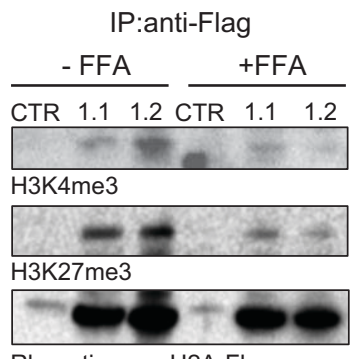

Rb: anti-macroH2A-Flag

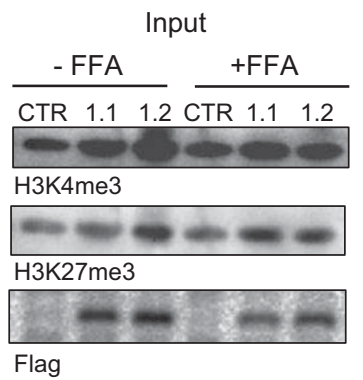

Figure 5. Western blot analysis and IP of epigenetic histone marks. A) Western blot analysis of FASN, SCD, H3K4me3, and H3K27me3 in simacroH2A1 HepG2 \pm FFA and after reoverexpression of macroH2A1.2. B) IP of macroH2A1 isoforms \pm FFA from HepG2-transfected cells. IP using anti-Flag was blotted with anti-H3K4me3 and anti-H3K27me3 and reblot (Rb) with antiFlag. Right panel shows input for anti-Flag, anti-H3K4me3, and anti-H3K27me3.

immunoprecipitated (Re-ChIP). The simultaneous presence of macroH2A1 isoforms and H3K27me3 on the promoter of SCD has been measured (Fig. 6B). The results suggest that the association of macroH2A1.1 isoform on the SCD promoter is strongly correlated with the epigenetic mark (H3K27me3) independently of the presence of FFAs.

These observations suggest that the macroH2A1 isoforms differentially modulate the association of histone marks in the regulation of lipogenic genes.

\section{DISCUSSION}

Excessive caloric intake in the western diet over the last decade is responsible for the increased levels of obesity that is expected to double by 2025 (35), and obesity is the most significant single risk factor for the development of hepatic steatosis. From an epigenetic point of view, the excess intake of nutrients was shown to alter the expression of genes and their transcriptional activity by affecting histone methylation (36). The novel findings described in this study represent a new mechanism for explaining how posttranslational modifications of lipogenic genes could be modulated by macroH2A1 histone variants in hepatic steatosis. In an in vitro model of fat-induced steatosis, only the macroH2A1.2 variant is up-regulated compared with the macroH2A1.1, which was also confirmed in a previous observation by using a murine model of fat-induced hepatocellular carcinoma (16). However, the mechanisms underlying the effects of the 2 isoforms on the regulation of gene expression related to lipid intake have not yet been reported. By using an in vitro model of steatosis transiently expressing the 2 isoforms, we demonstrated that isoform 1.2 responds to the FFA stimuli by inducing lipid accumulation, triglyceride levels, and LPO. In contrast, macroH2A1.1 was identified as the isoform with the capability to protect hepatocytes from lipid accumulation and LPO damage induced by FFAs. Previous studies showed that macroH2A1 has a role in the regulation of gene expression. Furthermore, broad-based examination of gene expression in macroH2A1 knockout mouse livers has identified an increased expression level of a cluster of genes directly involved in fatty acid metabolism $(13,14)$. In addition, macroH2A1 knockout mice fed a high-fat diet resulted in decreased body weight and fat mass (37). In the present study, we found that the overexpression of macroH2A1.2 up-regulated genes involved in gluconeogenesis, glucose transporters, and genes involved in de novo lipogenesis and $\beta$-oxidation differently from macroH2A1.1. It is noteworthy that the up-regulation of lipogenic genes is strongly associated with the expression of macroH2A1. Indeed the absence of macroH2A1 prevented the expression of the lipogenic genes in the presence of exogenous FFAs. Taken together, these results suggest that fat uptake is not sufficient to modulate the lipogenic genes unless macroH2A1 is present. Moreover, the absence of Atp5a1 and Fam 73b, known to be targeted by total macroH2A1 (27), is correlated with the prevention of the development of hepatic steatosis in mice under a highfat diet. This might be due to an increased expression of the macroH2A1.1 isoform, reaffirming its protective role. The lean phenotype and the predominance of macroH2A1.1 suggests that an alternative pathway may be involved as a solution to bypass ATP deficiency and could play a new role for the control of mitochondrial function. However, this warrants further investigation to ascertain the precise role of this histone variant.

The important role of macroH2A1 in the transcriptional regulation has been previously reported to positively correlate with H3K27me3 and also negatively with marks for transcription including RNA polymerase II and histone H3K4me3 methylation. However, macroH2A1 can play a positive role in gene expression in a subset of genes when it is bound in the transcribed regions (34). In this study, we 

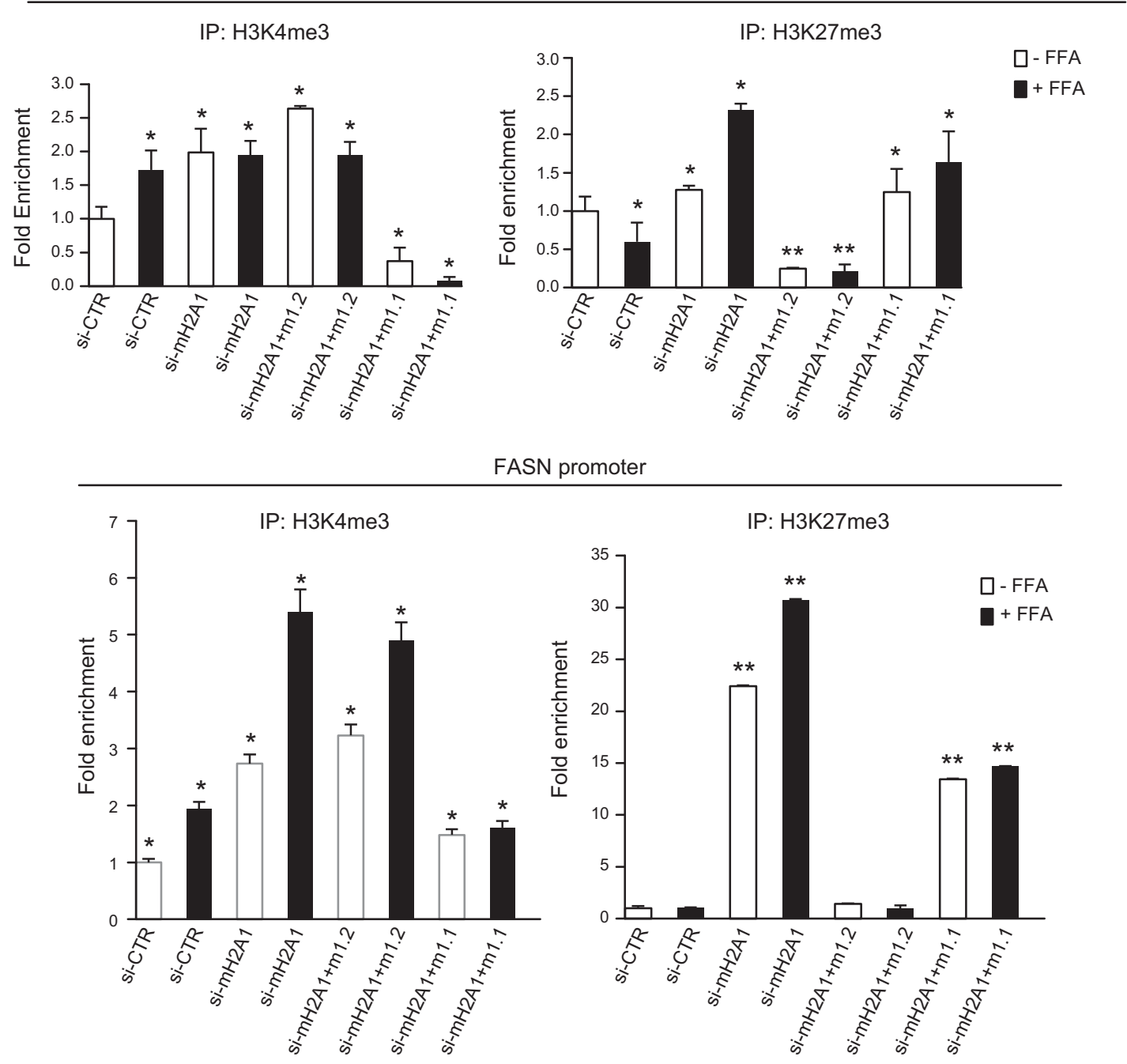

B

SCD promoter

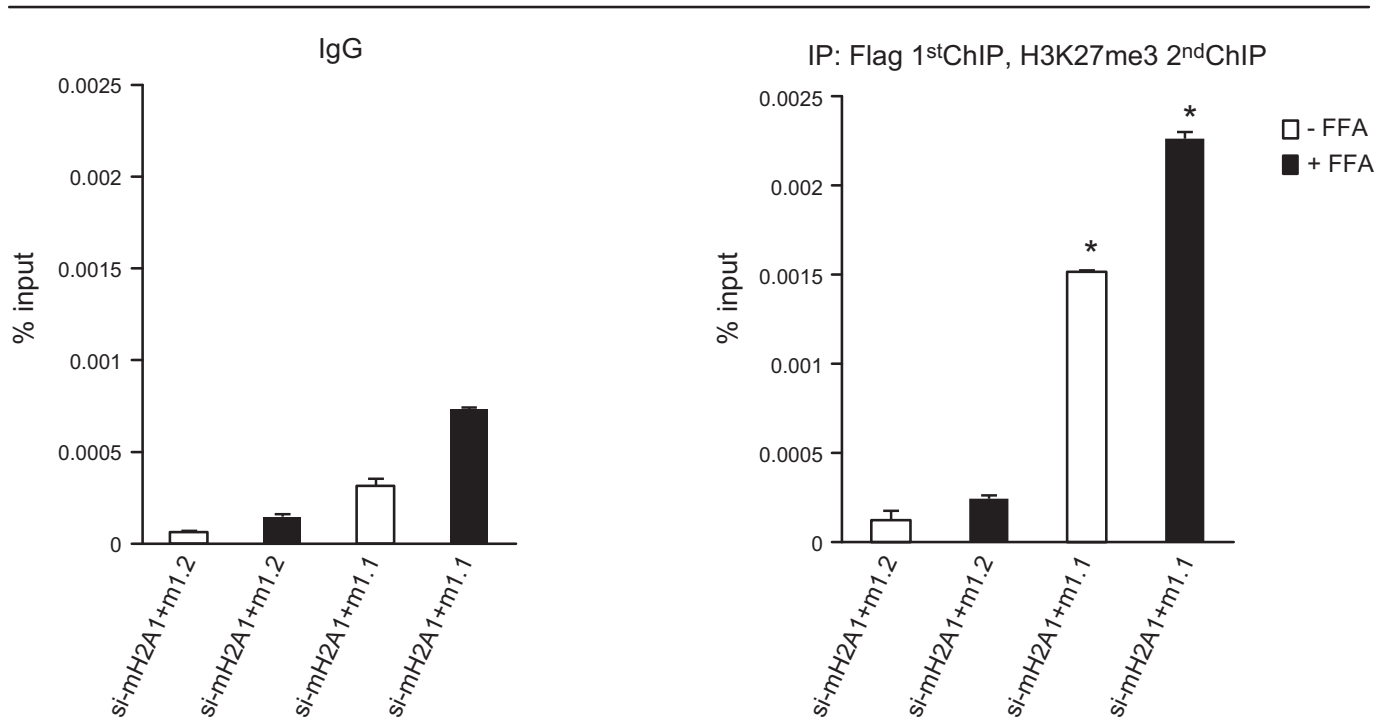

Figure 6. Recruitment of epigenetic histone marks on FASN and SCD promoters. A) HepG2 simacroH2A1 and reoverexpressing macroH2A1 isoforms \pm FFA were used for ChIP analysis with the indicated antibodies. Quantitative PCR was used to measure the level of enrichment on SCD and FASN promoters. Values of control were set at 1. Results represent fold enrichment after normalization with the DNA input. $B$ ) Re-ChIP analysis by sequential IP with Flag and H3K27me3 has been performed on SCD promoter. IgG was used as a control. The binding activity is given as a percentage of total input. Error bars represent SEM. $* P<0.05, * * P<0.01 ; n=3$. 
showed that the abrogation of macroH2A1 can affect the level of epigenetic marks (H3K27me3 and H3K4me3) on the promoter of specific lipogenic genes, FASN and SCD, and this can affect their expression even in the presence of FFAs. This suggests that macroH2A1 may have a dynamic role in the modulation of histone methylation status of the promoters of specific genes. In particular, the regulation of FASN and SCD has been associated with environmental factors such as nutritional conditions that can affect histone methylation in liver. The methylation status of these lipogenic genes can suppress their expression even in the presence of SREBF1, which is the specific transcription factor of SCD and FASN (38). Interestingly, our results show that the absence of macroH2A1 can induce high level of both active (H3K27me3) and repressive (H3K4me3) marks causing a bivalent state. The bivalent chromatin is defined by the presence of both activating and repressive histone modifications (39), also observed in Ppar $\gamma$ and Fabp7 promoters, a key regulator of adipogenesis and fatty acid uptake, respectively (40). The bivalent state maintains chromatin in a condition with the potential for rapid gene activation (41). Indeed, the bivalent state is resolved toward an active or a repressed state in the presence of macroH2A1.2 or macroH2A1.1, respectively.

In conclusion, this study is the first to demonstrate that macroH2A1 isoform expression, influenced by fat uptake, can differentially regulate lipid genes by modulating their level of histone methylation. The antagonistic role of macroH2A1 isoforms in chromatin conformational changes lead to the activation or repression of lipid genes, which in turn affect the development of hepatic steatosis. The protective role of macroH2A1.1 needs further investigation to understand the molecular mechanisms behind its overexpression in relation to which nutrients could induce its activation.

This study is the first detailed analysis of the macroH2A1 isoforms in the regulation of lipogenic genes by histone methylation, thereby leading to better understanding of the molecular mechanism underlying the epigenetic regulation of lipogenic genes and thus of hepatic steatosis.

The authors thank the staff from the Wellcome Trust Sanger Institute Mouse Genetics Project, Research Support Facility, and Mouse Informatics Group for excellent technical support. The authors also thank the histology support of Dr. Susan Newbigging and Lily Morikawa from the CMHD Pathology Core (www.cmhd.ca). This work was supported by the Institute of Hepatology, Foundation for Liver Research, London, United Kingdom.

\section{REFERENCES}

1. Feldstein, A. E. (2010) Novel insights into the pathophysiology of nonalcoholic fatty liver disease. Semin. Liver Dis. 30, 391-401

2. Browning, J. D., Szczepaniak, L. S., Dobbins, R., Nuremberg, P., Horton, J. D., Cohen, J. C., Grundy, S. M., and Hobbs, H. H. (2004) Prevalence of hepatic steatosis in an urban population in the United States: impact of ethnicity. Hepatology 40, 1387-1395

3. Mach, T. (2000) Fatty liver-current look at the old disease. Med. Sci. Monit. 6, 209-216

4. Angulo, P. (2002) Nonalcoholic fatty liver disease. N. Engl. J. Med. 346, 1221-1231

5. McClain, C. J., Mokshagundam, S. P., Barve, S. S., Song, Z., Hill, D. B., Chen, T., and Deaciuc, I. (2004) Mechanisms of nonalcoholic steatohepatitis. Alcohol 34, 67-79
6. Postic, C., and Girard, J. (2008) Contribution of de novo fatty acid synthesis to hepatic steatosis and insulin resistance: lessons from genetically engineered mice. J. Clin. Invest. 118, 829-838

7. Stefan, N., Kantartzis, K., and Häring, H. U. (2008) Causes and metabolic consequences of Fatty liver. Endocr. Rev. 29, 939-960

8. Turner, B. M. (2012) The adjustable nucleosome: an epigenetic signaling module. Trends Genet. 28, 436-444

9. Volle, C., and Dalal, Y. (2014) Histone variants: the tricksters of the chromatin world. Curr. Opin. Genet. Dev. 25, 8-14,138

10. Pehrson, J. R., and Fried, V. A. (1992) MacroH2A, a core histone containing a large nonhistone region. Science 257, 1398-1400

11. Pehrson, J. R., Costanzi, C., and Dharia, C. (1997) Developmental and tissue expression patterns of histone macroH2A1 subtypes. J. Cell. Biochem. 65, 107-113

12. Kustatscher, G., Hothorn, M., Pugieux, C., Scheffzek, K., and Ladurner, A. G. (2005) Splicing regulates NAD metabolite binding to histone macroH2A. Nat. Struct. Mol. Biol. 12, 624-625

13. Changolkar, L. N., Costanzi, C., Leu, N. A., Chen, D., McLaughlin, K. J., and Pehrson, J. R. (2007) Developmental changes in histone macroH2Al-mediated gene regulation. Mol. Cell. Biol. 27, 2758-2764

14. Gamble, M. J., and Kraus, W. L. (2010) Multiple facets of the unique histone variant macroH2A: from genomics to cell biology. Cell Cycle 9, 2568-2574

15. Pogribny, I. P., Tryndyak, V. P., Bagnyukova, T. V., Melnyk, S., Montgomery, B., Ross, S. A., Latendresse, J. R., Rusyn, I., and Beland, F. A. (2009) Hepatic epigenetic phenotype predetermines individual susceptibility to hepatic steatosis in mice fed a lipogenic methyl-deficient diet. J. Hepatol. 51, 176-186

16. Rappa, F., Greco, A., Podrini, C., Cappello, F., Foti, M., Bourgoin, L., Peyrou, M., Marino, A., Scibetta, N., Williams, R., Mazzoccoli, G., Federici, M., Pazienza, V., and Vinciguerra, M. (2013) Immunopositivity for histone macroH2A1 isoforms marks steatosis-associated hepatocellular carcinoma. PLoS ONE 8 e54458

17. Nguyen, T. H., Mai, G., Villiger, P., Oberholzer, J., Salmon, P., Morel, P., Bühler, L., and Trono, D. (2005) Treatment of acetaminophen-induced acute liver failure in the mouse with conditionally immortalized human hepatocytes. J. Hepatol. 43, 1031-1037

18. De Gottardi, A., Vinciguerra, M., Sgroi, A., Moukil, M., Ravier-Dall'Antonia, F., Pazienza, V., Pugnale, P., Foti, M., and Hadengue, A. (2007) Microarray analyses and molecular profiling of steatosis induction in immortalized human hepatocytes. Lab. Invest. 87, 792-806

19. Novikov, L., Park, J. W., Chen, H., Klerman, H., Jalloh, A. S., and Gamble, M. J. (2011) QKI-mediated alternative splicing of the histone variant MacroH2A1 regulates cancer cell proliferation. Mol. Cell. Biol. 31, 4244-4255

20. Cui, W., Chen, S. L., and Hu, K. Q. (2010) Quantification and mechanisms of oleic acid-induced steatosis in HepG2 cells. Am J Transl Res 2, 95-104

21. Skarnes, W. C., Rosen, B., West, A. P., Koutsourakis, M., Bushell, W., Iyer, V., Mujica, A. O., Thomas, M., Harrow, J., Cox, T., Jackson, D., Severin, J., Biggs, P., Fu, J., Nefedov, M., de Jong, P. J., Stewart, A. F., and Bradley, A. (2011) A conditional knockout resource for the genome-wide study of mouse gene function. Nature 474, 337-342

22. White, J. K., Gerdin, A. K., Karp, N. A., Ryder, E., Buljan, M., Bussell, J. N., Salisbury, J., Clare, S., Ingham, N. J., Podrini, C., Houghton, R., Estabel, J., Bottomley, J. R., Melvin, D. G., Sunter, D., Adams, N. C., Tannahill, D., Logan, D. W., Macarthur, D. G., Flint, J., Mahajan, V. B., Tsang, S. H., Smyth, I., Watt, F. M., Skarnes, W. C., Dougan, G., Adams, D. J., Ramirez-Solis, R., Bradley, A., and Steel, K. P.; Sanger Institute Mouse Genetics Project. (2013) Genome-wide generation and systematic phenotyping of knockout mice reveals new roles for many genes. Cell 154, 452-464

23. Ryder, E., Gleeson, D., Sethi, D., Vyas, S., Miklejewska, E., Dalvi, P., Habib, B., Cook, R., Hardy, M., Jhaveri, K., Bottomley, J., Wardle-Jones, H., Bussell, J. N., Houghton, R., Salisbury, J., Skarnes, W. C., and Ramirez-Solis, R.; Sanger Mouse Genetics Project. (2013) Molecular characterization of mutant mouse strains generated from the EUCOMM/KOMP-CSD ES cell resource. Mamm. Genome 24, 286-294

24. Adissu, H. A., Estabel, J., Sunter, D., Tuck, E., Hooks, Y., Carragher, D. M., Clarke, K., Karp, N. A., Newbigging, S., Jones, 
N., Morikawa, L., White, J. K., and McKerlie, C.; Sanger Mouse Genetics Project. (2014) Histopathology reveals correlative and unique phenotypes in a high-throughput mouse phenotyping screen. Dis. Model. Mech. 7, 515-524

25. Ricchi, M., Odoardi, M. R., Carulli, L., Anzivino, C., Ballestri, S., Pinetti, A., Fantoni, L. I., Marra, F., Bertolotti, M., Banni, S., Lonardo, A., Carulli, N., and Loria, P. (2009) Differential effect of oleic and palmitic acid on lipid accumulation and apoptosis in cultured hepatocytes. J. Gastroenterol. Hepatol. 24, 830-840

26. Repa, J. J., Liang, G., Ou, J., Bashmakov, Y., Lobaccaro, J. M., Shimomura, I., Shan, B., Brown, M. S., Goldstein, J. L., and Mangelsdorf, D. J. (2000) Regulation of mouse sterol regulatory element-binding protein-1c gene (SREBP-1c) by oxysterol receptors, LXRalpha and LXRbeta. Genes Dev. 14, 2819-2830

27. Changolkar, L. N., Singh, G., Cui, K., Berletch, J. B., Zhao, K., Disteche, C. M., and Pehrson, J. R. (2010) Genome-wide distribution of macroH2A1 histone variants in mouse liver chromatin. Mol. Cell. Biol. 30, 5473-5483

28. Assem, M., Schuetz, E. G., Leggas, M., Sun, D., Yasuda, K., Reid, G., Zelcer, N., Adachi, M., Strom, S., Evans, R. M., Moore, D. D., Borst, P., and Schuetz, J. D. (2004) Interactions between hepatic Mrp4 and Sult2a as revealed by the constitutive androstane receptor and Mrp4 knockout mice. J. Biol. Chem. 279, 2225022257

29. Halleck, M. S., Lawler JF, J. R., Blackshaw, S., Gao, L., Nagarajan, P., Hacker, C., Pyle, S., Newman, J. T., Nakanishi, Y., Ando, H., Weinstock, D., Williamson, P., and Schlegel, R. A. (1999) Differential expression of putative transbilayer amphipath transporters. Physiol. Genomics 1, 139-150

30. Kent, W. J., Sugnet, C. W., Furey, T. S., Roskin, K. M., Pringle, T. H., Zahler, A. M., and Haussler, D. (2002) The human genome browser at UCSC. Genome Res. 12, 996-1006

31. Kataoka, H., and Biswas, C. (1991) Nucleotide sequence of a cDNA for the alpha subunit of human mitochondrial ATP synthase. Biochim. Biophys. Acta 1089, 393-395

32. Bassett, J. H. D., Gogakos, A., White, J. K., Evans, H., Jacques, R. M., van der Spek, A. H., Ramirez-Solis, R., Ryder, E., Sunter, D., Boyde, A., Campbell, M. J., Croucher, P. I., and Williams, G. R.; Sanger Mouse Genetics Project. (2012) Rapid-throughput skeletal phenotyping of 100 knockout mice identifies 9 new genes that determine bone strength. PLoS Genet. 8, e1002858

33. Tappenden, D. M., Lynn, S. G., Crawford, R. B., Lee, K., Vengellur, A., Kaminski, N. E., Thomas, R. S., and LaPres, J. J. (2011) The aryl hydrocarbon receptor interacts with ATP5 $\alpha 1$, a subunit of the ATP synthase complex, and modulates mitochondrial function. Toxicol. Appl. Pharmacol. 254, 299-310

34. Gamble, M. J., Frizzell, K. M., Yang, C., Krishnakumar, R., and Kraus, W. L. (2010) The histone variant macroH2A1 marks repressed autosomal chromatin, but protects a subset of its target genes from silencing. Genes Dev. 24, 21-32

35. World Health Organization (1998) The World Health Report 1998. Life in the $21^{\text {st }}$ Century: A Vision for All, WHO, Geneva, Switzerland

36. Ghoshal, K., Li, X., Datta, J., Bai, S., Pogribny, I., Pogribny, M., Huang, Y., Young, D., and Jacob, S. T. (2006) A folate- and methyldeficient diet alters the expression of DNA methyltransferases and methyl CpG binding proteins involved in epigenetic gene silencing in livers of F344 rats. J. Nutr. 136, 1522-1527

37. Sheedfar, F., Vermeer, M., Pazienza, V., Villarroya, J., Rappa, F., Cappello, F., Mazzoccoli, G., Villarroya, F., Van der Molen, H., Hofker, M. H., Koonen, D. P., and Vinciguerra, M. (2014) Genetic ablation of macrohistone H2A1 leads to increased leanness, glucose tolerance and energy expenditure in mice fed a high-fat diet [Epub ahead of print]. Int. J. Obes. 10.1038/ijo.2014.91

38. Ehara, T., Kamei, Y., Takahashi, M., Yuan, X., Kanai, S., Tamura, E., Tanaka, M., Yamazaki, T., Miura, S., Ezaki, O., Suganami, T., Okano, M., and Ogawa, Y. (2012) Role of DNA methylation in the regulation of lipogenic glycerol-3-phosphate acyltransferase 1 gene expression in the mouse neonatal liver. Diabetes 61, 2442-2450

39. Lesch, B. J., Dokshin, G. A., Young, R. A., McCarrey, J. R., and Page, D. C. (2013) A set of genes critical to development is epigenetically poised in mouse germ cells from fetal stages through completion of meiosis. Proc. Natl. Acad. Sci. USA 110, 16061-16066

40. Mikkelsen, T. S., Ku, M., Jaffe, D. B., Issac, B., Lieberman, E., Giannoukos, G., Alvarez, P., Brockman, W., Kim, T. K., Koche, R. P., Lee, W., Mendenhall, E., O'Donovan, A., Presser, A., Russ, C., Xie, X., Meissner, A., Wernig, M., Jaenisch, R., Nusbaum, C., Lander, E. S., and Bernstein, B. E. (2007) Genome-wide maps of chromatin state in pluripotent and lineage-committed cells. $\mathrm{Na}$ ture $448,553-560$

41. Bernstein, B. E., Mikkelsen, T. S., Xie, X., Kamal, M., Huebert, D. J., Cuff, J., Fry, B., Meissner, A., Wernig, M., Plath, K., Jaenisch, R., Wagschal, A., Feil, R., Schreiber, S. L., and Lander, E. S. (2006) A bivalent chromatin structure marks key developmental genes in embryonic stem cells. Cell 125, 315-326

Received for publication August 20, 2014. Accepted for publication November 24, 2014. 